\title{
The total artificial heart in patients with congenital heart disease
}

\author{
Karthik Thangappan ${ }^{1}$, Awais Ashfaq ${ }^{1}$, Chet Villa ${ }^{2}$, David L. S. Morales ${ }^{1}$ \\ ${ }^{1}$ Department of Cardiothoracic Surgery, Cincinnati Children's Hospital Medical Center, Cincinnati, Ohio, USA; ${ }^{2}$ Department of Cardiology, \\ Cincinnati Children's Hospital Medical Center, Cincinnati, Ohio, USA \\ Correspondence to: David L. S. Morales, MD. Department of Cardiothoracic Surgery, Cincinnati Children's Hospital Medical Center, 3333 Burnet \\ Ave, MLC 2004, Cincinnati, OH 45229, USA. Email: David.Morales@cchmc.org.
}

\begin{abstract}
Background: While ventricular assist devices (VADs) remain the cornerstone of mechanical circulatory support (MCS), the total artificial heart (TAH-t) has gained popularity for certain patients in whom VAD support is not ideal. Congenital heart disease (CHD) patients often have barriers to VAD placement due to anatomic and physiological variation and thus can benefit from the TAH-t. The purpose of this study is to analyze the differences in TAH application and outcomes in patients with and without CHD.

Methods: The SynCardia Department of Clinical Research provided data upon request for all TAH- $t$ implantations worldwide from December 1985 to October 2019. These patients were divided into two groups by pre-implantation diagnosis of CHD and non-CHD.

Results: A total of 1,876 patients were identified. Eighty (4\%) of these patients also carried a diagnosis of CHD. There was a higher proportion of children in the CHD cohort $(16.3 \%$ vs. $2.1 \%, \mathrm{P}<0.001)$ and this translated into a lower average age amongst the two groups $(34 \pm 13$ vs. $49 \pm 13$ years, $\mathrm{P}<0.001)$. There were also significantly more females in the CHD group (22.8\% vs. $12.8 \%, \mathrm{P}=0.010)$. CHD patients were more likely to be supported with a $50 \mathrm{cc}$ TAH-t $(11.3 \%$ vs. $4.5 \%, \mathrm{P}=0.005)$ while all other support characteristics, including duration of support, were similar between the groups. All measured outcomes were similar between CHD and non-CHD patients including positive outcome (alive on device or transplanted), 1-month conditional survival, and rate of Freedom Driver use.

Conclusions: TAH-t is an effective means to support patients with CHD. Patients with CHD had similar survival, support characteristics, and frequency of discharge compared to patients without CHD. As MCS continues to grow, its indications broadened, and its contraindications narrowed, more patient populations will see the benefit of the TAH's continuously developing technology.
\end{abstract}

Keywords: Total artificial heart (TAH); congenital heart disease (CHD); heart transplantation; heart failure

Submitted Dec 30, 2019. Accepted for publication Jan 29, 2020.

doi: 10.21037 /acs.2020.02.08

View this article at: http://dx.doi.org/10.21037/acs.2020.02.08

\section{Introduction}

Mechanical circulatory support (MCS) is a standard of care in the adult cardiac surgery world and has been so for decades with improvements in mortality and quality of care for adults with refractory heart failure (1). Mechanical support has undergone tremendous growth and development over the years, now including both ventricular assist devices (VADs) and total artificial hearts (TAHs) in its arsenal.

MCS use, however, has not been limited to adults.
According to the United Network for Organ Sharing (UNOS) database for thoracic transplantation, in the last decade over 1,000 pediatric patients have been successfully bridged to heart transplantation using MCS. As the use of MCS has risen tremendously over the last decade, clear improvements in waitlist mortality have been shown both in children and adults $(1,2)$. However, the majority of children and adults supported over the last two decades carried a diagnosis of cardiomyopathy. Patients with congenital heart disease (CHD) across the age spectrum are less likely to receive MCS for end-stage heart failure and those awaiting 
transplantation are more likely to experience waitlist mortality as a result $(2,3)$.

The hesitancy to adopt MCS in these patients is likely due to a combination of factors such as patient size, complex anatomy, and higher incidence of end-organ dysfunction including hepatic, renal, and pulmonary systems as a consequence of both systolic and diastolic dysfunction (4-8). In the most recent years, over $40 \%$ of children have been supported to transplantation with MCS (9). The number of adults supported with a VAD or temporary total artificial heart (TAH-t) in the INTERMACS database has also quadrupled since 2010 (10). The increasing frequency of MCS in CHD patients reflects growing maturation of the field as well as the steady increase in the number and acuity of heart failure admissions in children and adults with CHD. There has been a two-fold increase in the number of advanced heart failure admissions in CHD patients since 2002 , and $16 \%$ of teenagers admitted with heart failure will experience mortality (11). The number of heart failure admissions for adults with CHD has grown by more than $90 \%$ over the last two decades (12). Adults with CHD currently make up $\sim 10 \%$ of heart transplantation registrants 18-39 years of age in the International Society for Heart and Lung Transplantation (ISHLT). Thus, it is clear that the number of patients with CHD who require MCS will continue to grow in the coming years (13).

The TAH-t is an integral part of the MCS support strategy for CHD patients who are not good candidates for systemic VAD therapy alone due to anatomic or physiologic considerations. The TAH-t has undergone much development in design since it hit the market in the 1980s. While the three companies SynCardia, AbioCor, and CARMAT all have contributed to the TAH-t field, only the SynCardia device has had a clinical impact with nearly 2,000 implants. TAH-t was first employed in adults with heart failure who could not be well-supported with a VAD or BiVAD. Eventually, its advantages for certain types of heart failure with specific etiologies (e.g., restrictive cardiomyopathy, biventricular failure, post-MI ventricular septal defect, primary arrhythmia) were realized. It also did not take long for the device to be expanded to pediatric patients, adolescents, and adults with CHD $(14,15)$. The device is especially useful in CHD patients with residual anatomic lesions that would complicate VAD therapy (16). Historically, one of the major factors limiting the use of the TAH-t was the size of the $70 \mathrm{cc}$ device. The development of the $50 \mathrm{cc}$ TAH-t and 3D virtual implantation has significantly increased the number of patients with small body surface area who may benefit from the device (notably women and children) $(17,18)$.

There are few studies investigating the outcomes of the TAH-t in CHD patients, mostly in review articles or center-specific case reports/series. This study sought to investigate the outcomes for CHD patients supported with the TAH-t and to compare these outcomes to non-CHD patients who underwent TAH-t implantation.

\section{Methods}

\section{Data collection}

SynCardia, Inc. retains records of all TAH-t implantations and patient characteristics. The SynCardia Department of Clinical Research provided the desired data using their company database. Between December 1985 and October 2019, 1,876 cases of TAH-t implantation have occurred using the SynCardia device worldwide. Data from all of these cases was obtained from SynCardia, Inc. for the purposes of this research article and was retrospectively analyzed. The total number of TAH-t cases was then stratified by pre-implantation diagnosis of CHD yielding two patient groups: CHD (congenital or genetic structural heart disease) and non-CHD (e.g., cardiomyopathy). Pediatric patients were defined as $<19$ years of age. Adult patients were defined as $\geq 19$ years of age (Figure 1).

\section{Statistical analysis}

Patient demographics, pre-implantation diagnoses, TAH-t support details (e.g., duration of support), and postoperative outcomes were listed for both CHD and non-CHD groups and then retrospectively analyzed to identify differences between the two groups. Categorical variables between the CHD and non-CHD groups were compared using Chisquare analysis. Mean values and standard deviation were compared using independent $t$-test. A $\mathrm{P}$ value of less than 0.05 was considered to be significant.

\section{Results}

\section{Demographics}

A total of 1,876 patients were implanted from December 1985 to October 2019. Eighty (4\%) of these patients also carried a diagnosis of CHD (Table 1). There was a higher proportion of children in the CHD cohort $(16.3 \%$ vs. $2.1 \%, \mathrm{P}<0.001)$ and this translated into a lower average age 


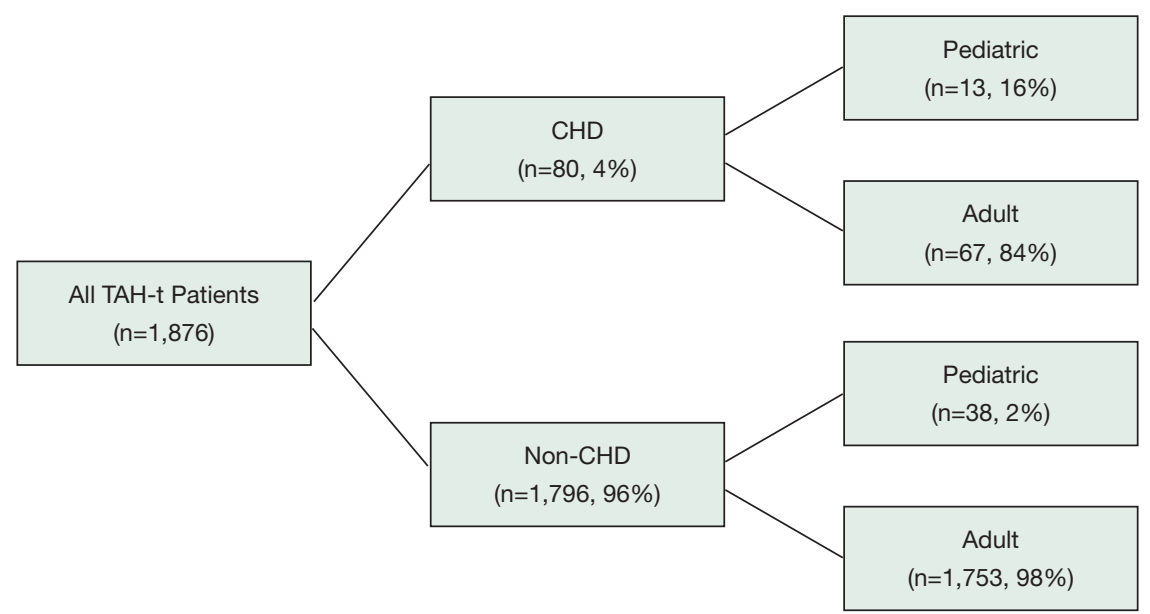

Figure 1 Division of patient groups and respective age distribution ( $\mathrm{n}=3$ patients with unknown age data in non-CHD group not-included in age distribution).

amongst the two groups $(34 \pm 13$ vs. $49 \pm 13$ years, $\mathrm{P}<0.001)$. There were also significantly more females in the CHD group (22.8\% vs. $12.8 \%, \mathrm{P}=0.010)$. As expected, there were significantly more ischemic and dilated cardiomyopathy patients in the non-CHD group than in the CHD group $(\mathrm{P}<0.001)$. All other listed pre-implantation diagnoses including restrictive cardiomyopathy, valvular cardiomyopathy, and infectious complications were similar between the two groups.

\section{TAH-t support analysis}

CHD patients were more likely to be supported with a 50 cc TAH-t $(11.3 \%$ vs. $4.5 \%, \mathrm{P}=0.005)$ while all other support characteristics were similar between the groups (Table 2). The average number of days on support was similar between the groups when analyzed in a continuous manner or in 6-month blocks $(145 \pm 222$ vs. $136 \pm 212$ days, $\mathrm{P}=0.711)$.

\section{Outcomes}

All measured outcomes were similar between CHD and non-CHD patients (Table 3). Positive outcome was defined as transplanted or alive on device. Just over $50 \%$ of patients were successfully transplanted in each group. There were no differences found between the two groups regarding the following outcomes: positive outcome (56.3\% vs. $53.0 \%$, $\mathrm{P}=0.574)$ and died on device $(43.8 \%$ vs. $47.0 \%, \mathrm{P}=0.574)$. The same holds true when patients were stratified by age (Table 3). Outcomes measured at two months postimplantation were also similar between the groups. Over $20 \%$ of patients in each group were transplanted within 30 days of TAH-t implantation $(\mathrm{P}=0.827)$. Total mortality between the groups both at 2-days $(17.1 \%$ vs. $10.0 \%$, $\mathrm{P}=0.175)$ and 1 -month post-implantation $(62.9 \%$ vs. $52.3 \%$, $\mathrm{P}=0.220)$ were similar, as was 1 -month conditional survival ( $72.5 \%$ vs. $75.8 \%, \mathrm{P}=0.502)$ with conditional survival meaning exclusion of patients who did not survive at least 1 month.

Statistics regarding the growing use of the Freedom Driver (FD) was also analyzed. Over $20 \%$ of patients in each group were transferred over to the FD. Average time from implant to transfer was similar between the groups ( $65 \pm 64$ vs. $62 \pm 91$ days, $\mathrm{P}=0.884$ ). Over $60 \%$ of patients in each group who received the FD were able to be discharged home $(65.0 \%$ vs. $64.2 \%, \mathrm{P}=0.944)$. Approximately $70 \%$ of patients in each group were transplanted after transfer to the FD $(70.0 \%$ vs. $69.2 \%, \mathrm{P}=0.939)$.

\section{Discussion}

The last decade has seen an increase in the number of patients with CHD and heart failure as well as the use of MCS in these patients. The TAH-t has the potential to effectively support patients with CHD when VAD therapy alone is not possible due to residual anatomic or physiologic abnormalities (19). For example, TAH-t is a more ideal choice when multiple residual defects are present in combination with heart failure such as a stenotic pulmonary 


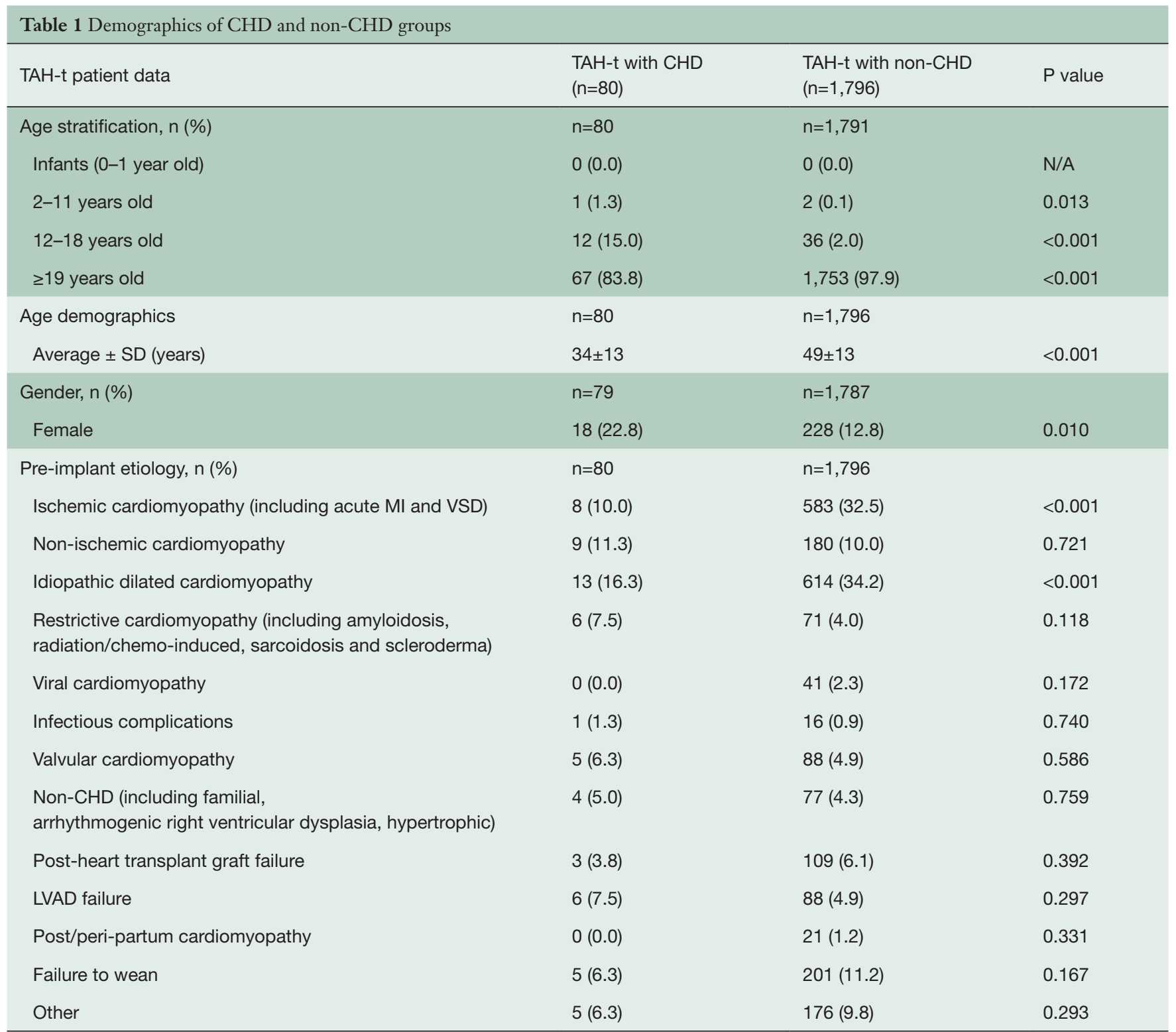

conduit, aortic insufficiency, and a ventricular septal defect, since these defects would need to be corrected prior to VAD implantation. In contrast, these defects are resected en bloc during the TAH-t implantation. Furthermore, CHD often manifests with restrictive cardiac physiology which is not ideal for VAD therapy. The Fontan circulation, due to systemic-pulmonary collateral formation and resulting subphysiological cardiac output and high central venous pressure, often has detrimental effects on end-organ function. As the source of abnormalities in these patients is the single-ventricle anatomy and its venous congestive nature, resecting this and replacing it with a TAH-t would restore biventricular physiology and most importantly, create a normal or low central venous pressure, which will be key to recovering many chronically failing end-organs.

This is the first large-scale analysis of the TAH-t outcomes for patients with and without CHD in the current era in which both the 50 and 70 cc devices are available. There were expected differences in patient demographics between groups as the CHD patients were significantly younger, smaller, and more likely to be female. These findings are consistent with the evolving epidemiology of 


\begin{tabular}{|c|c|c|c|}
\hline \multicolumn{4}{|l|}{ TAH-t patient data, n (\%) } \\
\hline $50 \mathrm{cc}$ TAH-t & $9(11.3)$ & $80(4.5)$ & 0.005 \\
\hline 70 cc TAH-t & $71(88.8)$ & $1,716(95.9)$ & 0.002 \\
\hline Total days (all patients, years) & $11,554(31.6)$ & $243,441(667.0)$ & $N / A$ \\
\hline Average \pm SD (days) & $145 \pm 222$ & $136 \pm 212$ & 0.711 \\
\hline Duration, n (\%) & $\mathrm{n}=80$ & $\mathrm{n}=1,789$ & \\
\hline 0-6 months & $60(75.0)$ & 1,393 (77.9) & 0.547 \\
\hline$>24$ months & $3(3.8)$ & $48(2.7)$ & 0.567 \\
\hline
\end{tabular}

Percentages based on number of patients in each group with available data for given variable as indicated by $n=x x$ listed in same row as variable name.

CHD as well as the persistent differences in the frequency of support use amongst adult men and women with dilated cardiomyopathy $(20,21)$.

We defined positive outcome as transplanted or alive on device. The current study demonstrates that patients with CHD have similar outcomes including discharge, time to transplantation, and incidence of positive outcome when compared to patients without CHD. The survival outcomes for each group are notable given that the overall survival in the current study parallels recent data from IMACS and EuroMACS $(22,23)$. The similar outcomes are also worth noting, given that CHD is a significant predictor for outcomes in the larger cohort of patients requiring MCS (23). When analyzing outcomes in the last ten years compared to the preceding 25 years, it is first notable that the rate of TAH implantation in both CHD and nonCHD patients has increased dramatically over time. While outcomes are not significantly improved in the most recent ten years, this is likely a reflection of the expansion of TAH use to more heterogeneous and sicker patient populations. Although the outcomes are overall similar between the two groups, it is worth noting that conditional 1-month survival is superior to overall survival for either group. In other words, survival of patients who survived at least 1 month post-implantation is much better than survival of all patients. When patients do not survive in the immediate months following implantation, this is more often than not a reflection of poor patient selection. Given that the majority of the mortality hazard for patients requiring biventricular support is within the first 30-60 days after implantation, earlier implantation and revised implantation guidelines are likely to improve patient condition at implantation and overall outcomes $(22,23)$.

Patient selection and indications for earlier implantation are especially important given the variety of anatomic and physiologic characteristics specific to heart failure in patients with CHD. The TAH-t has been used to support patients with univentricular as well biventricular circulation (24). TAH support should be considered as a superior biventricular bridge to transplantation as outcomes are more favorable than biventricular VAD support $(25,26)$. Moreover, TAH-t allows providers to offer treatment to certain patient cohorts who are not well supported with VAD therapy such as certain CHD patients, those patients with chronic heart transplant rejection, chronic right ventricular failure with an LVAD in place, biventricular failure, cardiac tumors, intractable arrhythmias, patients with active malignancy, restrictive physiology, or those with significant end-organ dysfunction secondary to heart failure (27). With the aging population of CHD into adulthood, more patients will develop heart failure requiring alternative treatments to transplantation.

Recently, there has also been an increase in the number of implants with the $70 \mathrm{cc}$ TAH-t device (28). This is likely 


\begin{tabular}{|c|c|c|c|}
\hline Variables & $\begin{array}{l}\text { TAH-t with CHD } \\
(n=80)\end{array}$ & $\begin{array}{l}\text { TAH-t with non-CHD } \\
(n=1,789)\end{array}$ & $P$ value \\
\hline Transplant & $41(51.3)$ & $903(50.5)$ & 0.892 \\
\hline Alive on device & $4(5.0)$ & $46(2.6)$ & 0.188 \\
\hline Outcomes <19 years old, n (\%) & $n=13$ & $\mathrm{n}=38$ & \\
\hline Transplant & $10(76.9)$ & $25(65.8)$ & 0.455 \\
\hline Alive on device & $0(0.0)$ & $1(2.6)$ & 0.555 \\
\hline Alive on device & $4(6.0)$ & $45(2.6)$ & 0.092 \\
\hline Died on device & $32(47.8)$ & $828(47.3)$ & 0.939 \\
\hline Overall survival conditional on 1 month (30 days), $\mathrm{n}(\%)$ & $\mathrm{n}=80$ & $\mathrm{n}=1,789$ & \\
\hline Overall survival conditional on 1 month (30 days) & $58(72.5)$ & $1,356(75.8)$ & 0.502 \\
\hline Total mortality, n (\%) & $\mathrm{n}=35$ & $n=828$ & \\
\hline Within 30 days & $22(62.9)$ & $433(52.3)$ & 0.220 \\
\hline Within 2 days & $6(17.1)$ & $83(10.0)$ & 0.175 \\
\hline Transplant & $28(47.5)$ & $455(44.4)$ & 0.645 \\
\hline Alive on device & $4(6.8)$ & $46(4.5)$ & 0.414 \\
\hline Died on device & $27(45.8)$ & $524(51.1)$ & 0.423 \\
\hline Outcomes [1985-2009], n (\%) & $n=21$ & $n=764$ & \\
\hline Transplant & $13(61.9)$ & $448(58.1)$ & 0.764 \\
\hline Alive on device & $0(0.0)$ & $0(0.0)$ & $\mathrm{N} / \mathrm{A}$ \\
\hline Died on device & $8(38.1)$ & $316(41.4)$ & 0.764 \\
\hline Freedom driver support, n (\%) & $n=80$ & $\mathrm{n}=1,796$ & \\
\hline Number of patients transferred & $20(25.0)$ & $383(21.3)$ & 0.434 \\
\hline
\end{tabular}

Table 3 (continued) 


\begin{tabular}{|c|c|c|c|}
\hline Average duration from implant to transfer (days) [range] & $65 \pm 64[20-311]$ & $62 \pm 91[0-1,253]$ & 0.884 \\
\hline Number of patients discharged, $n(\%)$ & $13(65.0)$ & $246(64.2)$ & 0.944 \\
\hline Total freedom duration, all patients, days (years) & $5,748(15.7)$ & $104,208(285.5)$ & $\mathrm{N} / \mathrm{A}$ \\
\hline Average $\pm \mathrm{SD}$ (days) & $287 \pm 321$ & $272 \pm 316$ & 0.836 \\
\hline Freedom driver survival, $\mathrm{n}(\%)$ & $\mathrm{n}=20$ & $\mathrm{n}=383$ & \\
\hline
\end{tabular}

multifactorial but is in part due to the FDA approval of the SynCardia Freedom Driver in 2010 and more use of this device in patients with right ventricular failure after LVAD placement. The Freedom Driver dramatically improves portability and maneuverability, allowing patients to be discharged from the hospital while the device is in place. It is clearly seen that there was no difference between the groups who had the Freedom Driver in terms of support duration, survival to transplantation, and mortality.

The current report in conjunction with the availability of the smaller $50 \mathrm{cc}$ device suggests that what were once considered anatomic contraindications to TAH-t use may need to be reconsidered. Just over $10 \%$ of patients with $\mathrm{CHD}$ received the $50 \mathrm{cc}$ device, suggesting the device is being employed more often in the current era. Furthermore, novel implantation approaches, in conjunction with virtual implantation will likely only broaden the population of smaller and more complex CHD patients, especially those with the Fontan circulation, who may benefit from the device $(18,29)$.

\section{Limitations}

While this study contains data referring to the worldwide experience of the SynCardia TAH-t, it has certain noteworthy limitations. These include the retrospective nature of the study as well as the pre-consolidated nature of the data provided. Raw patient and device data are not available from SynCardiaSystems, LLC, so further statistical analysis past Chi-Square and $t$-test comparisons (e.g., Kaplan-Meier survival, Cox-regression hazard ratios) was not possible which limited the depth of statistical analysis we were able to conduct. In addition, data regarding complications was not available, limiting analysis of the post-operative outcomes.

\section{Conclusions}

The current study demonstrates that the TAH-t is an effective means to support patients with CHD. Patients with CHD had similar survival and frequency of discharge compared to patients without CHD. Furthermore, this study continues to demonstrate the utility of the $50 \mathrm{cc}$ device in supporting underserved populations, including those with a smaller chest cavity such as younger patients and women. As MCS continues to grow, its indications broadened, and its contraindications narrowed, more patient populations will see the benefit of this continuously developing technology. With the current trends in frequency of use and outcomes of both VAD and TAH-t as well as the constant trajectory of technology, MCS as a whole will most likely become, in the coming decades, the standard of care for heart failure secondary to not only cardiomyopathy, but also CHD. 


\section{Acknowledgments}

Special thanks to Breeana McCombs, Judy Skroback, and Brock Vendsel from SynCardia Systems, LLC for their help with data collection.

\section{Footnote}

Conflicts of Interest: Dr. Morales discloses the following: consultant and member of medical advisory board for Berlin Heart, Inc; proctor, consultant, and member of medical advisory board for SynCardia Systems, LLC; national primary investigator for the 50/50 cc SynCardia Total Artificial Heart Food and Drug Administration trial; consultant for Medtronic, Inc (HeartWare Division); consultant for Abbott Medical, Inc (Thoratec Division); and consultant, investigator, and member of medical advisory board for CorMatrix Cardiovascular, Inc. Drs. Thangappan, Ashfaq, and Villa have nothing to disclose with regard to commercial support.

\section{References}

1. Emin A, Rogers CA, Parameshwar J, et al. Trends in longterm mechanical circulatory support for advanced heart failure in the UK. Eur J Heart Fail 2013;15:1185-93.

2. Zafar F, Castleberry C, Khan MS, et al. Pediatric heart transplant waiting list mortality in the era of ventricular assist devices. J Heart Lung Transplant 2015;34:82-8.

3. Everitt MD, Donaldson AE, Stehlik J, et al. Would access to device therapies improve transplant outcomes for adults with congenital heart disease? Analysis of the United Network for Organ Sharing (UNOS). J Heart Lung Transplant 2011;30:395-401.

4. Kiesewetter CH, Sheron N, Vettukattill JJ, et al. Hepatic changes in the failing Fontan circulation. Heart 2007;93:579-84.

5. Dimopoulos K, Diller GP, Koltsida E, et al. Prevalence, predictors, and prognostic value of renal dysfunction in adults with congenital heart disease. Circulation 2008;117:2320-8.

6. Ridderbos FJ, Wolff D, Timmer A, et al. Adverse pulmonary vascular remodeling in the Fontan circulation. J Heart Lung Transplant 2015;34:404-13.

7. Piran S, Veldtman G, Siu S, et al. Heart failure and ventricular dysfunction in patients with single or systemic right ventricles. Circulation 2002;105:1189-94.

8. Gewillig M, Daenen W, Aubert A, et al. Abolishment of chronic volume overload. Implications for diastolic function of the systemic ventricle immediately after Fontan repair. Circulation 1992;86:II93-9.

9. Shugh SB, Riggs KW, Morales DLS. Mechanical circulatory support in children: past, present and future. Transl Pediatr 2019;8:269-77.

10. Cedars A, Vanderpluym C, Koehl D, et al. An Interagency Registry for Mechanically Assisted Circulatory Support (INTERMACS) analysis of hospitalization, functional status, and mortality after mechanical circulatory support in adults with congenital heart disease. J Heart Lung Transplant 2018;37:619-30.

11. Burstein DS, Shamszad P, Dai D, et al. Significant mortality, morbidity and resource utilization associated with advanced heart failure in congenital heart disease in children and young adults. Am Heart J 2019;209:9-19.

12. Burchill LJ, Gao L, Kovacs AH, et al. Hospitalization Trends and Health Resource Use for Adult Congenital Heart Disease-Related Heart Failure. J Am Heart Assoc 2018;7:e008775.

13. Dipchand AI, Edwards LB, Kucheryavaya AY, et al. The registry of the International Society for Heart and Lung Transplantation: seventeenth official pediatric heart transplantation report--2014; focus theme: retransplantation. J Heart Lung Transplant 2014;33:985-95.

14. Ryan TD, Jefferies JL, Zafar F, et al. The evolving role of the total artificial heart in the management of endstage congenital heart disease and adolescents. ASAIO J 2015;61:8-14.

15. Morales DL, Khan MS, Gottlieb EA, et al. Implantation of total artificial heart in congenital heart disease. Semin Thorac Cardiovasc Surg 2012;24:142-3.

16. Villa CR, Morales DLS. The Total Artificial Heart in End-Stage Congenital Heart Disease. Front Physiol 2017;8:131.

17. Wells D, Villa CR, Simón Morales DL. The 50/50 cc Total Artificial Heart Trial: Extending the Benefits of the Total Artificial Heart to Underserved Populations. Semin Thorac Cardiovasc Surg Pediatr Card Surg Annu 2017;20:16-9.

18. Moore RA, Lorts A, Madueme PC, et al. Virtual implantation of the 50cc SynCardia total artificial heart. J Heart Lung Transplant 2016;35:824-7.

19. Adachi I, Morales DS. Implantation of total artificial heart in congenital heart disease. J Vis Exp 2014. doi: 10.3791/51569.

20. Gilboa SM, Devine OJ, Kucik JE, et al. Congenital 
Heart Defects in the United States: Estimating the Magnitude of the Affected Population in 2010. Circulation 2016;134:101-9.

21. Hsich EM, Naftel DC, Myers SL, et al. Should women receive left ventricular assist device support?: findings from INTERMACS. Circ Heart Fail 2012;5:234-40.

22. Vierecke J, Gahl B, de By TMMH, et al. Results of primary biventricular support: an analysis of data from the EUROMACS registry. Eur J Cardiothorac Surg 2019;56:1037-45.

23. Kirklin JK, Xie R, Cowger J, et al. Second annual report from the ISHLT Mechanically Assisted Circulatory Support Registry. J Heart Lung Transplant 2018;37:685-91.

24. Rossano JW, Goldberg DJ, Fuller S, et al. Successful use of the total artificial heart in the failing Fontan circulation. Ann Thorac Surg 2014;97:1438-40.

25. Nguyen A, Pozzi M, Mastroianni C, et al. Bridge to transplantation using paracorporeal biventricular

Cite this article as: Thangappan K, Ashfaq A, Villa C, Morales DLS. The total artificial heart in patients with congenital heart disease. Ann Cardiothorac Surg 2020;9(2):89-97. doi: 10.21037/ acs.2020.02.08 assist devices or the syncardia temporary total artificial heart: is there a difference? J Cardiovasc Surg (Torino) 2015;56:493-502.

26. Arabia F, Gregoric I, Kasirajan V, et al. Total artificial heart (TAH): survival outcomes, risk factors, adverse events in Intermacs. J Heart Lung Transplant 2016;35:S95.

27. Ryan TD, Jefferies JL, Zafar F, Angela L, Morales DLS. The evolving role of Total Artificial Heart in the management of end stage congenital heart disease and adolescents. ASAIO J 2015;61:8-14.

28. Kirklin JK, Naftel DC, Pagani FD, et al. Seventh INTERMACS annual report: 15,000 patients and counting. J Heart Lung Transplant 2015;34:1495-504.

29. Woods RK, Kindel S, Mitchell ME, et al. Evolving understanding of total artificial heart support of young infants and children. J Thorac Cardiovasc Surg 2020;159:1075-82. 